\section{Ultrasonographic echogenicity of normal salivary glands in adults: comparison of submandibular and parotid glands}

Insik Choi, Dong Gyu Na, Wooyul Paik

Department of Radiology, Gangneung Asan Hospital, University of Ulsan College of Medicine, Gangneung, Korea

Purpose: The normal submandibular gland (SMG) and parotid gland (PG) are thought to have similar homogeneous ultrasound (US) hyperechogenicity; however, this has not been extensively investigated. The aim of this study was to determine whether the normal SMG and PG have similar US echogenicity.

Methods: We included 969 consecutive adult patients with normal salivary glands. The patients were categorized into three age groups: group 1 (19 to 29 years, $n=27$ ), group 2 (30 to 49 years, $n=273$ ), and group 3 ( $\geq 50$ years, $n=669$ ). The echogenicities of the SMG and PG were prospectively evaluated by an experienced radiologist. Computed tomography (CT) attenuation in Hounsfield units (HUs) was quantitatively measured for the SMG, PG, and sternocleidomastoid muscle in 140 patients.

Results: Relative to the PG, the echogenicity of the SMG was similar in $706(73.0 \%)$ and homogeneously hypoechoic in 263 patients (27.0\%). The frequency of SMG hypoechogenicity decreased with increasing age (group 1, 59.3\%; group 2, 36.3\%; group 3, 22.1\%; $P<0.001$ ). The CT attenuation levels (in HUs) of the SMG and PG were significantly higher in patients with hypoechoic SMGs than in patients with SMG echogenicity similar to that of the $P G(P<0.001)$.

Conclusion: Hypoechogenicity of the SMG was observed in more than a quarter of this sample of adults with normal salivary glands. The SMG may be inadequate as a reference standard for evaluating thyroid nodule echogenicity in patients with diffuse thyroid disease with decreased echogenicity.

Keywords: Submandibular gland; Parotid gland; Thyroid gland; Ultrasonography

\section{Introduction}

The major salivary glands, including the parotid gland (PG) and submandibular gland (SMG), normally exhibit homogeneous and hyperechoic ultrasound (US) echogenicity relative to the adjacent neck muscles [1,2]. Although Katz et al. [3] reported that the SMG was more hypoechoic than the PG, the normal PG and SMG have been generally thought to have homogeneous hyperechogenicity comparable to the echogenicity of the normal thyroid gland $[4,5]$. Thyroid nodule echogenicity is an important US feature for predicting the risk of malignancy [6-8]. The current US guidelines

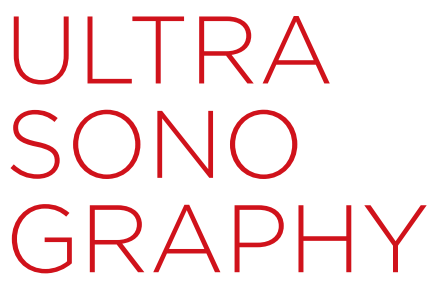

ORIGINAL ARTICLE

https://doi.org/10.14366/usg.20070 pISSN: 2288-5919 - elSSN: 2288-5943

Ultrasonography 2021;40:342-348

Received: May 20, 2020

Revised: August 11, 2020

Accepted: August 21, 2020

Correspondence to:

Dong Gyu Na, MD, PhD, Department

of Radiology, Gangneung Asan

Hospital, University of Ulsan College

of Medicine, 38 Bangdong-gil,

Gangneung 25440, Korea

Tel. $+82-33-610-4310$

Fax. +82-33-610-3490

E-mail: nndgna@gmail.com

This is an Open Access article distributed under the terms of the Creative Commons Attribution NonCommercial License (http://creativecommons.org/ licenses/by-nc/4.0/) which permits unrestricted noncommercial use, distribution, and reproduction in any medium, provided the original work is properly cited.

Copyright @ 2021 Korean Society of Ultrasound in Medicine (KSUM)

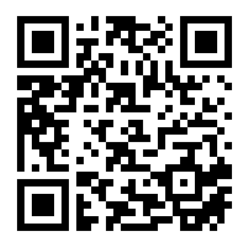

How to cite this article:

Choi I, Na DG, Paik W. Ultrasonographic echogenicity of normal salivary glands in adults: comparison of submandibular and parotid glands. Ultrasonography. 2021 Jul;40(3):342-348. 
for nodular thyroid disease recommend that the echogenicity of thyroid nodules be described in comparison with the anterior neck muscles and the surrounding thyroid parenchyma [6-8]. However, in patients with underlying diffuse thyroid disease with decreased parenchymal echogenicity, assessment of the nodule echogenicity can be inaccurate and problematic. When the surrounding thyroid parenchymal echogenicity is abnormally low, some thyroid US guidelines recommend that the echogenicity of thyroid nodules be described in comparison to the strap muscles and abnormal surrounding thyroid tissue on US reports $[6,7]$. Meanwhile, some European US guidelines have proposed that the SMG could be an alternative reference for evaluating the echogenicity of thyroid nodules in cases of decreased echogenicity of the surrounding thyroid parenchyma $[8,9]$. This latter guideline is based on the empirical observation that the normal echogenicity of the SMG is similar to that of the thyroid gland. However, this assumption is not proven and requires further research.

Although most agree that the major salivary glands have similar US echogenicity, no study has compared the echogenicity of the normal PG and SMG, and the conventional understanding of this relationship lacks evidence. Therefore, the purpose of this study was to determine whether the normal SMG and PG have similar US echogenicity. We also sought to investigate the relationships of SMG echogenicity with age, sex, and computed tomography (CT) attenuation.

\section{Materials and Methods}

This retrospective study was approved by the institutional review board of Gangneung Asan Hospital (GNAH 2020-2-009), and the requirement for informed consent was waived.

\section{Patients}

Between April 2017 and November 2017, 1245 consecutive adult patients underwent neck US for thyroid nodules $(n=925)$, postoperative surveillance of thyroid cancer $(n=209)$, diffuse thyroid disease or goiter $(n=42)$, lymphadenopathy $(n=33)$, neck mass $(n=13)$, salivary gland disease $(n=13)$, hyperparathyroidism $(n=3)$, neck discomfort $(n=3)$, voice change $(n=3)$, and deep neck infection $(n=1)$. In this study, we included patients with normal salivary glands, defined as persons who had no clinical history of salivary disease and no suspicion of salivary gland disease on clinical and US assessment. We excluded 276 patients after reviewing their electronic medical records and performing a prospective assessment of the US features of the salivary glands. The exclusion criteria were as follows: patients who had received radioactive iodine therapy after thyroidectomy due to thyroid cancer $(n=207)$, patients with salivary gland tumors $(n=14)$, patients with suspected chronic sialadenitis showing bilateral diffuse heterogeneous echogenicity of the $P G$ or SMG regardless of clinical features $(n=13)$, patients with unilateral focal or diffuse hypoechogenicity of the PG or SMG $(n=12)$, patients with symmetrical homogeneous mild hypoechogenicity of the bilateral PG regardless of clinical features $(n=8)$, patients who underwent radiation therapy on the neck due to head and neck cancer $(n=6)$, patients with periglandular foreign bodies with severe posterior acoustic shadowing $(n=2)$, patients with a deep neck infection around the salivary glands $(n=1)$, and patients in whom prospective US evaluation of the salivary glands was not conducted $(n=13)$ during the study period. The PG was considered normal for this study only if the bilateral PGs showed symmetrical homogeneous hyperechogenicity and lacked clinical suspicion of salivary gland disease. Regarding the SMG, bilateral symmetrical homogeneous hypoechogenicity was considered indicative of a normal SMG if the patient had no clinical history or symptoms of salivary gland disease and no other coexisting US abnormalities, including heterogeneous echogenicity, focal parenchymal lesions, sialectasia or sialolithiasis, and glandular enlargement or atrophic change of the SMG.

Following the exclusion of 276 patients, our study population comprised 969 patients (784 women, 185 men; median age, 57 years; interquartile range, 40 to 74 years). The 969 patients were categorized by age into three groups (group 1, 19-29 years; group 2, 30-49 years; group 3, $\geq 50$ years). Neck CT scans were performed within 3 years before or after neck US in 140 of the 969 patients $(14.4 \%)$ for the evaluation of thyroid tumors $(n=120)$, lymphadenopathy $(n=10)$, neck masses $(n=6)$, goiter $(n=3)$, and hoarseness $(n=1)$.

\section{US Examination and Image Analysis}

All US examinations were performed with a 5- to $12-\mathrm{MHz}$ lineararray transducer (EPIQ7, Philips Healthcare, Bothell, WA, USA), and the US echogenicity of the salivary glands was prospectively evaluated by one experienced radiologist (D.G.N.) with 22 years of experience performing thyroid and neck US. Both the PG and SMG were examined with the patient in a supine position with the head turned to the contralateral side. We assessed the echogenicity of both the PG and the SMG with the same US scan parameters, including the level of gain, the focal zone, and the application of compound imaging (SonoCT). We compared the echogenicity of the SMG to that of the superficial lobe of the ipsilateral PG and divided patients accordingly into an isoechoic group and a hypoechoic group. The isoechoic group included patients in whom the echogenicity of the SMG was similar to that of the PG, while the hypoechoic group contained patients in whom the echogenicity of 
the SMG was obviously hypoechoic compared to that of the PG (Figs. $1-3)$.

\section{CT Image Analysis}

CT scanning was performed with a single-slice CT scanner $(n=1)$, a 64-slice CT scanner ( $n=97)$, or a 128-section CT scanner $(n=42)$. The CT attenuation (in Hounsfield units [HU]) of the SMG and PG was quantitatively measured by a resident (I.C.) using a region of interest of $45-55 \mathrm{~mm}^{2}$ on the unenhanced axial CT images. We obtained the average attenuation (in HUs) on the ipsilateral superficial lobe of the $P G, S M G$, and sternocleidomastoid muscle (SCM). We measured the $\mathrm{HU}$ difference between the PG or SMG and the ipsilateral SCM by subtracting the value for the PG or SMG from that of the SCM, respectively (SCM-PG and SCM-SMG, respectively).

\section{Data Analysis and Statistics}

The continuous variables are presented as the mean \pm standard deviation or the median (interquartile range) based on a parametric or nonparametric distribution, respectively. The categorical variables are reported as frequencies and percentages for each category. The mean patient age was compared between the two SMG echogenicity groups (the isoechoic and hypoechoic group) using the Mann-Whitney $\mathrm{U}$ test. The chi-square test for trends was used to investigate the relationship between SMG echogenicity and age. The unpaired $t$ test was used to compare the attenuation (in HUs) of the PG, SMG, SCM-PG, and SCM-SMG between the two SMG echogenicity groups. Spearman correlation coefficients were calculated between age and attenuation of the PG and SMG. Statistical analysis was performed using SPSS version 23.0 for Windows (IBM Corp., Armonk, NY, USA), and a significant difference was indicated by a P-value $<0.05$.

\section{Results}

\section{Age and Sex of Patients according to US Echogenicity of the Normal SMG}

Relative to the echogenicity of the normal PG, the US echogenicity of the normal SMG was classified as isoechoic in $706(73.0 \%)$ and hypoechoic in 263 of the 969 patients (27.0\%). No significant difference in sex was observed between the two groups ( $P=0.598)$, and the patients in the isoechoic group were slightly older

Table 1. Age and sex of patients according to US echogenicity of the normal SMG

\begin{tabular}{llll}
\hline \multirow{2}{*}{ Patient data } & \multicolumn{2}{c}{ US echogenicity of the SMG ${ }^{\text {a) }}$} & \multirow{2}{*}{ P-value } \\
\cline { 2 - 3 } & \multicolumn{1}{c}{ Isoechoic } & Hypoechoic & \\
\hline No. of patients & $706(73.0)$ & $263(27.0)$ & \\
No. of women & $575(81.4)$ & $209(79.5)$ & 0.598 \\
Age (year) & $57.5(41.5-73.5)$ & $53.0(32-74)$ & $<0.001$ \\
\hline
\end{tabular}

Values are presented as number (\%) or median (interquartile range).

US, ultrasound; SMG, submandibular gland.

${ }^{a}$ The SMG was classified as isoechoic when its echogenicity was similar to that of the parotid gland and hypoechoic when its echogenicity was obviously lower than that of the parotid gland.

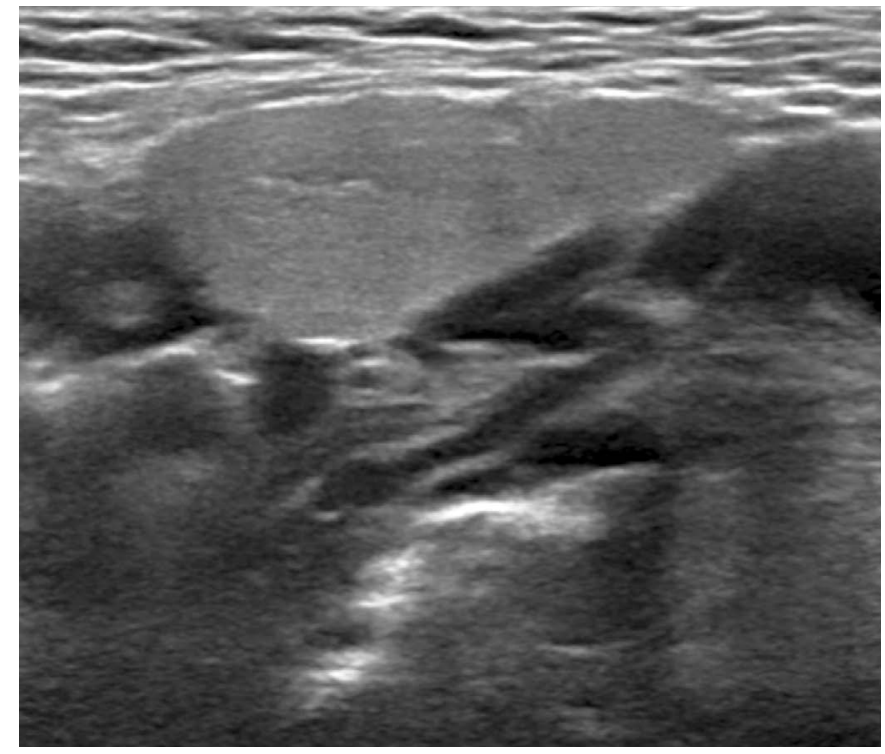

A

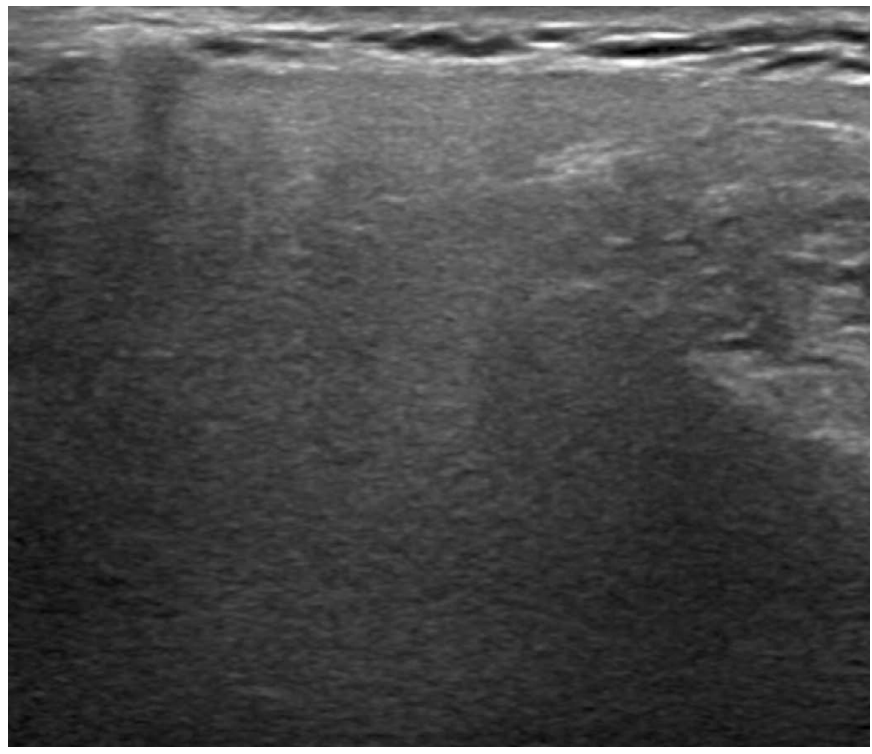

B

Fig. 1. A 52-year-old woman with an isoechoic normal submandibular gland compared to the normal parotid gland.

The right submandibular gland (A) shows homogeneous hyperechogenicity similar to the right parotid gland (B). 


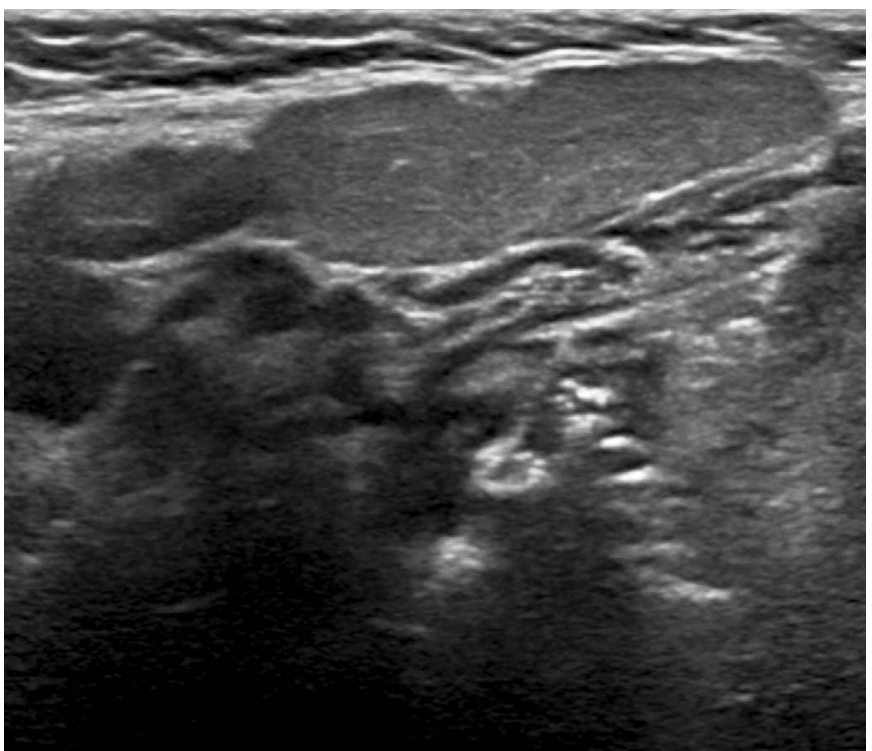

A

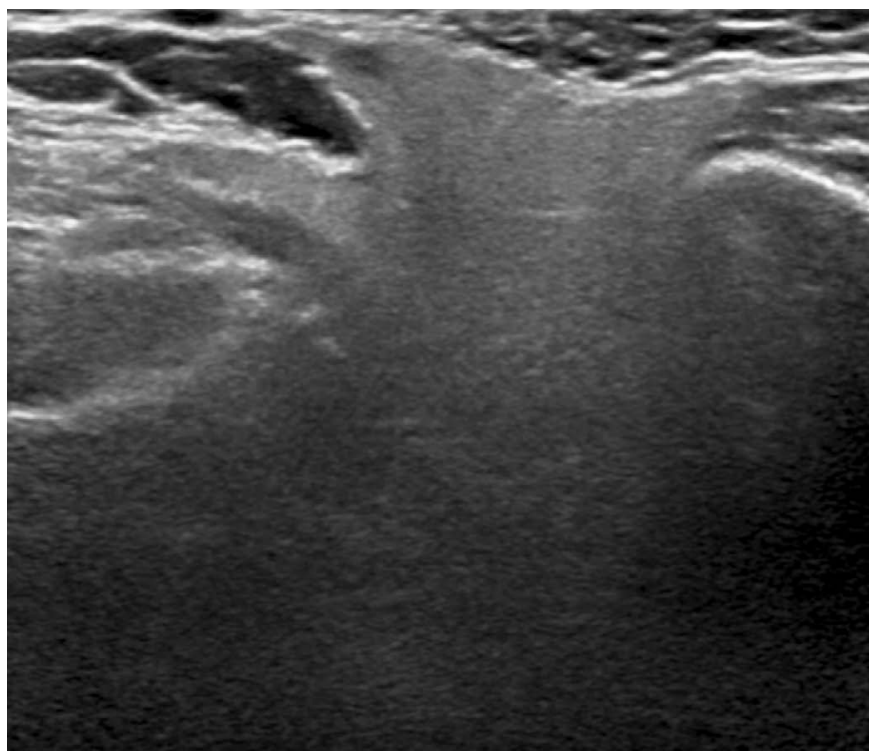

B

Fig. 2. A 60-year-old woman with a mildly hypoechoic normal submandibular gland compared to the normal parotid gland. The right submandibular gland (A) shows homogeneous mild hypoechogenicity compared to the right parotid gland (B).

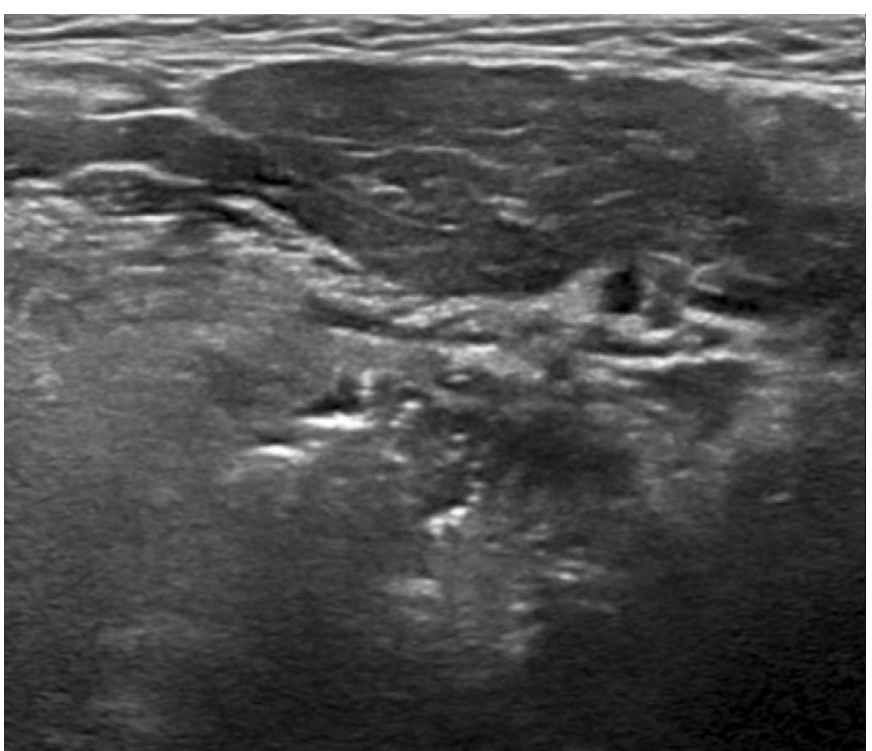

A

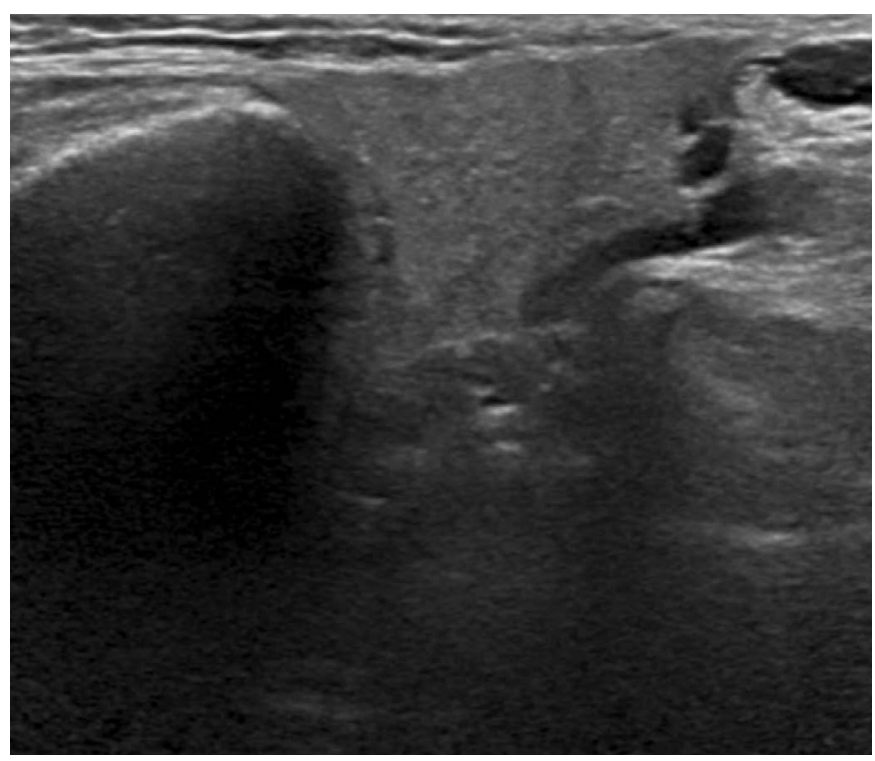

B

Fig. 3. A 28-year-old woman with a markedly hypoechoic normal submandibular gland compared to the normal parotid gland. The left submandibular gland (A) shows homogeneous marked hypoechogenicity compared to the left parotid gland (B).

than those in the hypoechoic group (57.5 years vs. 53.0 years, respectively; $\mathrm{P}<0.001$ ) (Table 1).

\section{US Echogenicity of the Normal SMG according to Age Group}

Of the 969 patients, $27(2.8 \%)$ were aged $19-29$ years, $273(28.2 \%)$ were aged 30-49 years, and 669 (69.0\%) were 50 years old or older. Hypoechogenicity of the SMG was found in 16 (59.3\%) of the patients $19-29$ years old, 99 (36.3\%) of the patients $30-49$ years old, and $148(22.1 \%)$ of the patients 50 years old or older. As age increased, the frequency of SMG hypoechogenicity tended to decrease $(P<0.001)$ (Table 2$)$. 
Table 2. Frequency of hypoechoic normal SMGs according to age group

\begin{tabular}{lcccc}
\hline \multirow{2}{*}{$\begin{array}{c}\text { SMG } \\
\text { echogenicity }\end{array}$} & $\begin{array}{c}\text { Group 1 } \\
(\mathrm{n}=27)\end{array}$ & $\begin{array}{c}\text { Group 2 } \\
(\mathrm{n}=273)\end{array}$ & $\begin{array}{c}\text { Group 3 } \\
(\mathrm{n}=669)\end{array}$ & P-value \\
\hline $\begin{array}{l}\text { Isoechoic } \\
(\mathrm{n}=706)\end{array}$ & $11(40.7)$ & $174(63.7)$ & $521(77.9)$ & $<0.001$ \\
$\begin{array}{l}\text { Hypoechoic } \\
(n=263)\end{array}$ & $16(59.3)$ & $99(36.3)$ & $148(22.1)$ & \\
\hline
\end{tabular}

Values are presented as number (\%).

SMG, submandibular gland.

${ }^{a}$ Group 1, 19-29 years; group 2, 30-49 years; group 3, $\geq 50$ years.

Table 3. US echogenicity and CT attenuation of normal SMG and PG

\begin{tabular}{lccc}
\hline \multirow{2}{*}{ CT data } & \multicolumn{2}{c}{ US echogenicity of the SMG $^{\text {a) }}$} & \multirow{2}{*}{ P-value } \\
\cline { 2 - 3 } & Isoechoic & Hypoechoic & \\
\hline No. of patients & $101(72.1)$ & $39(27.9)$ & \\
CT attenuation & & & \\
SMG (HU) & $35.2 \pm 12.0$ & $44.4 \pm 11.2$ & $<0.001$ \\
SCM-SMG (HU) & $17.0 \pm 13.7$ & $10.8 \pm 11.5$ & 0.013 \\
PG (HU) & $-32.9 \pm 21.5$ & $-16.5 \pm 18.9$ & $<0.001$ \\
SCM-PG (HU) & $85.1 \pm 24.9$ & $71.7 \pm 18.8$ & 0.003 \\
\hline
\end{tabular}

Values are presented as number (\%) or mean \pm standard deviation.

US, ultrasound; $C T$, computed tomography; SMG, submandibular gland; $P G$, parotid gland; HU, Hounsfield unit; SCM, sternocleidomastoid muscle.

${ }^{a}$ )The SMG was classified as isoechoic when its echogenicity was similar to that of the PG and hypoechoic when its echogenicity was lower than that of the PG.

\section{Associations among US Echogenicity, CT Attenuation, and Age}

Among 140 patients who underwent a neck CT scan, the SMG was isoechoic in 101 (72.1\%) and hypoechoic in 39 (27.9\%). The mean $\mathrm{CT}$ attenuation (in HUs) of the SMG was significantly higher than that of the PG in both the isoechoic (35.2 HU vs. $-32.9 \mathrm{HU}$, respectively; $\mathrm{P}<0.001)$ and hypoechoic groups (44.4 HU vs. -16.5 $H U$, respectively; $P<0.001)$. The mean $C T$ attenuation of the SMG was significantly lower in patients with isoechoic SMGs than that in patients with hypoechoic SMGs (35.2 HU vs. $44.4 \mathrm{HU}$, respectively; $P<0.001)$. The mean $C T$ attenuation of the $P G$ was significantly lower in patients with isoechoic SMGs than in those with hypoechoic SMGs ( $-32.9 \mathrm{HU}$ vs. $-16.5 \mathrm{HU}$, respectively; $\mathrm{P}<0.001)$. The mean CT attenuation levels of the SCM-SMG and SCM-PG were significantly higher in patients with isoechoic SMGs than in those with hypoechoic SMGs ( $P=0.013$ and $P=0.003$, respectively) (Table 3). The $C T$ attenuation levels of the $P G$ and $S M G$ showed significant negative correlations with age $(P G, r=-0.189, P=0.026 ; S M G, r=-$ $0.326, \mathrm{P}<0.001)$.

\section{Discussion}

Our study demonstrated that $27.0 \%$ of patients with no clinical or radiological evidence of salivary gland disease exhibited hypoechogenicity of the SMG relative to the PG. The frequency of SMG hypoechogenicity decreased with increasing age.

Our study results suggest two clinical implications. First, bilateral homogeneous hypoechogenicity of the SMG should not be mistaken for salivary gland disorders such as autoimmune sialadenitis (Sjögren syndrome) or IgG4-related disease if the case involves no clinical suspicion of sialadenitis and no other concurrent US abnormality of the PG and SMG. Although Sjögren syndrome or IgG4-related disease may cause hypoechogenicity of the SMG, in most cases this can be differentiated from physiologic hypoechogenicity of the normal SMG by the presence of a heterogeneous echotexture, nodular hypoechoic lesions, asymmetrical involvement, and glandular enlargement or atrophic change [10-13]. Second, the echogenicity of the normal SMG seems inappropriate as a reference standard for assessing the echogenicity of thyroid nodules in cases of thyroiditis with decreased parenchymal echogenicity because, based on our study results, the normal SMG may exhibit physiologic hypoechogenicity. The echogenicity of the normal PG may be more appropriate as a reference standard for evaluating the echogenicity of thyroid nodules in thyroiditis with decreased parenchymal echogenicity. This is because most of the normal PGs exhibited constant homogeneous hyperechogenicity in our study. However, our results require further investigation involving a comparison of the echogenicity of the normal PG and thyroid gland to evaluate the reliability of the normal PG as a reference structure with similar echogenicity to the normal thyroid gland. This study excluded eight patients who had mild hypoechogenicity of the PG relative to the normal thyroid gland from the study population, as our goal was to exclude patients with potential salivary gland disorders even when they had no clinical features of sialadenitis. Further investigation is required to determine whether the normal PG may show physiologic hypoechogenicity compared with the echogenicity of the normal thyroid gland.

Although the mechanism of physiologic hypoechogenicity of the normal SMG is uncertain, it may relate to normal variations in the amount of adipose tissue and the histologic architecture of salivary glandular tissue. It is well known that the normal PG has a greater amount of adipose tissue and a lower $\mathrm{CT}$ attenuation than the normal SMG [14]. This aligns with our findings that the CT attenuation of the normal PG was significantly lower than that of the normal SMG. Our study also showed that the CT attenuation of the hypoechoic SMG was higher than that of the isoechoic SMG, which suggests that the hypoechoic SMG may contain less fat tissue 
than the isoechoic SMG. Prior studies [14-22] have established that the amounts of adipose tissue and fibrous tissue in both the PG and the SMG tend to increase with age. These histologic changes may explain our findings of age-related changes in the CT attenuation of the PG and SMG. Notably, a prior study [14] reported a similar result, specifically that the $C T$ attenuation of the $P G$ and SMG decreased with increasing age. Therefore, the decrease in the frequency of SMG hypoechogenicity with increasing age in our study may also relate to the histologic changes in the normal salivary gland that occur with age. However, the difference in the amount of fat tissue between the PG and SMG cannot explain the similar echogenicity of the SMG and the $P G$, because the $C T$ attenuation of the SMG was substantially higher than that of the PG in patients who had similar echogenicity of the SMG and the PG (the isoechoic group). Fat tissue can be hypoechoic or hyperechoic depending on its heterogeneity [23]. PGs with complete fatty changes after radioactive iodine ablation for the treatment of thyroid cancer usually exhibit hypoechogenicity [24]. Tissue echogenicity is related to the acoustic reflection caused by variations in the acoustic impedance [25], and tissue with heterogeneous components may display hyperechogenicity due to the presence of multiple acoustic interfaces and increased acoustic reflections within the tissue. Therefore, the amount of adipose tissue does not appear to be the only factor that affects the echogenicity of the PG and SMG. Other histologic features, including heterogeneous architectures composed of acinar cells, excretory ducts, and connective tissue stroma with fibrous septa, may be related to the echogenicity of the salivary glands.

This study had several limitations. First, we could not determine the presence of normal variation in the echogenicity of the PG because we excluded all patients with decreased PG echogenicity, even if they were not suspected of having a salivary gland disorder. Second, pediatric patients were not included in this study, and the proportion of SMG hypoechogenicity in normal pediatric salivary glands therefore could not be determined. Third, we did not assess the interobserver agreement regarding the echogenicity of the salivary glands because only one experienced radiologist prospectively evaluated the US echogenicity of the salivary glands. A further prospective investigation of the interobserver agreement will be necessary to validate the reliability of our observations. Fourth, the number of young adult patients under 30 years was relatively small in our study population. Further investigation with a large population of young adults may be necessary to determine the prevalence of the hypoechoic normal SMG in young adults.

In conclusion, SMG hypoechogenicity was observed in more than a quarter of adults with normal salivary glands and was more frequently found in young adults than in older individuals. Bilaterally symmetrical, homogeneously hypoechoic SMGs without other glandular abnormalities should not be considered abnormal. The echogenicity of the SMG may be inadequate as a reference standard for evaluating thyroid nodule echogenicity in patients with diffuse thyroid disease and decreased parenchymal echogenicity.

ORCID: Insik Choi: https://orcid.org/0000-0001-7613-7976; Dong Gyu Na: https:// orcid.org/0000-0001-6422-1652; Wooyul Paik: https://orcid.org/0000-0001-96176227

\section{Author Contributions}

Conceptualization: Na DG. Data acquisition: Na DG, Choi I. Data analysis or interpretation: Na DG, Choi I. Drafting of the manuscript: Choi I. Critical revision of the manuscript: Na DG, Paik W. Approval of the final version of the manuscript: all authors.

\section{Conflict of Interest}

No potential conflict of interest relevant to this article was reported.

\section{References}

1. Gritzmann N, Rettenbacher T, Hollerweger $A$, Macheiner $P$, Hubner $E$. Sonography of the salivary glands. Eur Radiol 2003;13:964-975.

2. Bialek EJ, Jakubowski W, Zajkowski P, Szopinski KT, Osmolski A. US of the major salivary glands: anatomy and spatial relationships, pathologic conditions, and pitfalls. Radiographics 2006;26:745763.

3. Katz P, Hartl DM, Guerre A. Clinical ultrasound of the salivary glands. Otolaryngol Clin North Am 2009;42:973-1000.

4. Som PM, Curtin HD. Head and neck imaging. 5th ed. St. Louis, MO: Mosby Elsevier, 2011;2181-2202.

5. Zajkowski P, Ochal-Choinska A. Standards for the assessment of salivary glands: an update. J Ultrason 2016;16:175-190.

6. Grant EG, Tessler FN, Hoang JK, Langer JE, Beland MD, Berland LL, et al. Thyroid ultrasound reporting lexicon: white paper of the ACR Thyroid Imaging, Reporting and Data System (TIRADS) Committee. J Am Coll Radiol 2015;12:1272-1279.

7. Shin JH, Baek JH, Chung J, Ha EJ, Kim JH, Lee YH, et al. Ultrasonography diagnosis and imaging-based management of thyroid nodules: revised Korean Society of Thyroid Radiology Consensus Statement and Recommendations. Korean J Radiol 2016;17:370-395.

8. Russ G, Bonnema SJ, Erdogan MF, Durante C, Ngu R, Leenhardt L. European Thyroid Association guidelines for ultrasound malignancy risk stratification of thyroid nodules in adults: the EU-TIRADS. Eur Thyroid J 2017;6:225-237.

9. Rago T, Cantisani V, Ianni F, Chiovato L, Garberoglio R, Durante C, et al. Thyroid ultrasonography reporting: consensus of Italian Thyroid Association (AIT), Italian Society of Endocrinology (SIE), Italian 
Society of Ultrasonography in Medicine and Biology (SIUMB) and Ultrasound Chapter of Italian Society of Medical Radiology (SIRM). J Endocrinol Invest 2018;41:1435-1443.

10. Baldini C, Luciano N, Tarantini G, Pascale R, Sernissi F, Mosca M, et al. Salivary gland ultrasonography: a highly specific tool for the early diagnosis of primary Sjogren's syndrome. Arthritis Res Ther 2015;17:146.

11. Narayan AK, Baer A, Fradin J. Sonographic findings of IgG4-related disease of the salivary glands: case report and review of the literature. J Clin Ultrasound 2018;46:73-77.

12. Puxeddu I, Capecchi R, Carta F, Tavoni AG, Migliorini P, Puxeddu R. Salivary gland pathology in IgG4-related disease: a comprehensive review. J Immunol Res 2018;2018:6936727.

13. Martel A, Coiffier G, Bleuzen A, Goasguen J, de Bandt M, Deligny $C$, et al. What is the best salivary gland ultrasonography scoring methods for the diagnosis of primary or secondary Sjogren's syndromes? Joint Bone Spine 2019;86:211-217.

14. Mahne A, El-Haddad G, Alavi A, Houseni M, Moonis G, Mong A, et al. Assessment of age-related morphological and functional changes of selected structures of the head and neck by computed tomography, magnetic resonance imaging, and positron emission tomography. Semin Nucl Med 2007;37:88-102.

15. Andrew W. A comparison of age changes in salivary glands of man and of the rat. J Gerontol 1952;7:178-190.

16. Garrett JR. Some observations on human submandibular salivary glands. Proc R Soc Med 1962;55:488-491.

17. Garrett JR. The ultrastructure of intracellular fat in the parenchyma of human submandibular salivary glands. Arch Oral Biol 1963:8:729-734.

18. Scott J. Structure and function in aging human salivary glands. Gerodontology 1986;5:149-158.

19. Scott J, Flower EA, Burns J. A quantitative study of histological changes in the human parotid gland occurring with adult age. J Oral Pathol 1987;16:505-510.

20. Dayan D, Vered M, Paz T, Buchner A. Aging of human palatal salivary glands: a histomorphometric study. Exp Gerontol 2000;35:85-93.

21. Azevedo LR, Damante JH, Lara VS, Lauris JR. Age-related changes in human sublingual glands: a post mortem study. Arch Oral Biol 2005;50:565-574.

22. Garcia DS, Bussoloti Filho I. Fat deposition of parotid glands. Braz J Otorhinolaryngol 2013;79:173-176.

23. Spencer GM, Rubens DJ, Roach DJ. Hypoechoic fat: a sonographic pitfall. AJR Am J Roentgenol 1995;164:1277-1280.

24. Kim DW. Ultrasonographic features of the major salivary glands after radioactive iodine ablation in patients with papillary thyroid carcinoma. Ultrasound Med Biol 2015;41:2640-2645.

25. Rumack CM, Wilson SR, Charboneau JW. Diagnostic ultrasound. 3rd ed. St. Louis, MO: Mosby, 2005. 